\title{
At the root of brain cancer
}

Michael F. Clarke

\section{A small subpopulation of cells, 'brain-cancer stem cells', has been identified in humans. They have the exclusive ability to drive tumour formation, and could prove an effective target for therapies.}

$\mathrm{B}$ rain cancers are among the most devastating tumours in humans and are often rapidly fatal despite aggressive treatments. These tumours typically contain varied populations of cells that differ in the specific proteins - or markers - displayed on the cell surface.

On page 396 of this issue, Singh and colleagues ${ }^{1}$ describe how they have isolated a minority population of human brain-cancer cells based on the expression of a cell-surface marker called CD133. They report that, when injected into the brains of mice, this subpopulation of $\mathrm{CD}_{133^{+}}$cells could by itself drive tumour growth and dissemination. As few as one hundred of the CD133 ${ }^{+}$ cells formed tumours that could be serially transmitted from mouse to mouse, whereas tens of thousands of cancer cells lacking CD133 failed to do so. When tumours that arose from the injected CD $133^{+}$cells were examined, the cellular heterogeneity and architecture closely resembled that of the patients' tumours from which the cells had originally been taken. These findings add brain tumours to the list of cancers, including blood ${ }^{2-4}$ and epithelial cancers ${ }^{5}$, in which a cancer stem-cell population has been found.

Insights into how tissues are maintained provide hints as to why only a minority of the cancer cells drives tumour formation. Most cancers arise in tissues, such as the bone marrow, gut and breast, that are composed of a cellular hierarchy in which a small population of stem cells gives rise to progenitor cells that regenerate mature tissue cells. It was once thought that neuron formation in the brain was essentially complete by birth. However, recent findings demonstrate that the brain, like other organs in which cancers arise, contains a stem-cell population that can give rise to differentiated cells, including mature neurons.

In the normal brain, neuronal stem cells as well as early progenitor cells, but not their fully mature progeny, express the CD133 marker (Fig. 1a) ${ }^{6}$. In the brain tumours examined, Singh et al. ${ }^{1}$ found distinct subpopulations of cells that expressed either CD133 or various markers of mature brain cells. Thus, the cellular architecture of the brain tumours may be a caricature of that of the normal brain, with brain-cancer stem cells, probably derived from normal $\mathrm{CD}_{133^{+}}$stem or progenitor cells, giving rise

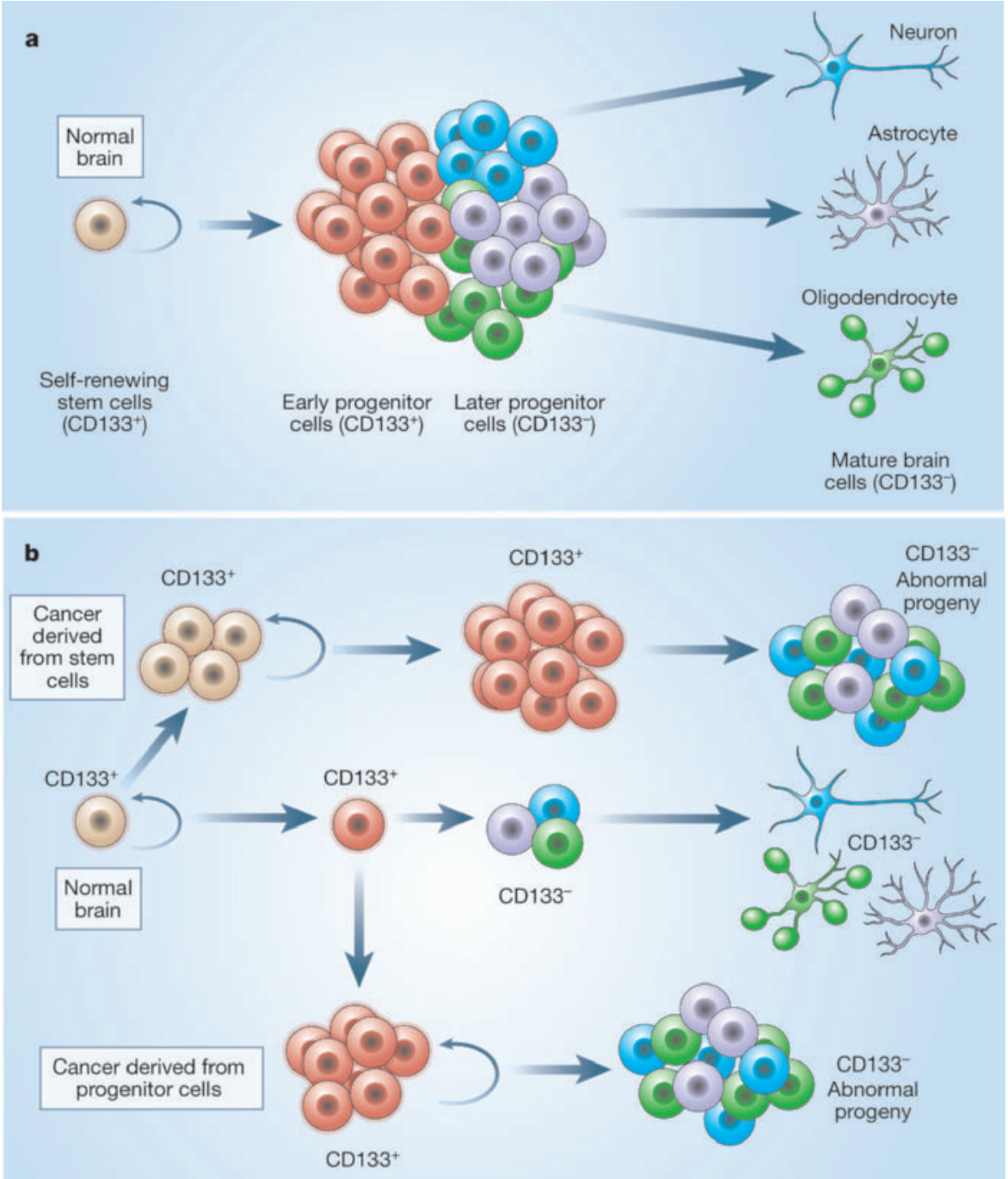

Figure 1 Brain-cell hierarchy. a, In the normal brain, stem cells, which express the CD133 marker and so are designated $\mathrm{CD}_{133^{+}}$, can generate new stem cells by the process of self-renewal. They can also produce early progenitor cells $\left(\mathrm{CD}_{133^{+}}\right)$, and later progenitor cells $\left(\mathrm{CD}_{133^{-}}\right)$that give rise to the mature forms of brain cells (neurons, astrocytes and oligodendrocytes, all $\mathrm{CD} 133^{-}$). Unlike the stem cells, progenitor cells have limited ability to replicate. b, Singh $e t$ al. ${ }^{1}$ have identified cancer stem cells. Such cells could arise from $\mathrm{CD}_{133^{+}}$brain stem cells, when loss of normal constraints results in expansion of the abnormal stem-cell pool, or from early $\mathrm{CD}_{133^{+}}$progenitor cells as a result of mutations that make these cells self-renewing. In either case, the cancer stem cells also generate abnormally differentiated $\mathrm{CD}_{133^{-}}$progeny that cannot self-renew and thus cannot form new tumours.

to aberrantly differentiated progeny (Fig. 1b).

Stem cells have two unique properties that make it likely that they are involved in cancer development. First, they are often the only long-lived cells in a tissue that have the ability to replicate. Typically, multiple mutations, occurring over many years, are necessary before a cell becomes cancerous. So the implication here is that cancer-inducing mutations accumulate in the long-lived, normal stem cells.

Second, through a process called selfrenewal, stem cells generate new stem cells with similar proliferation and differentiation capacities to their parental cell. By contrast, with each round of replication, 
progenitor cells become progressively more differentiated and are eventually destined to stop proliferating. Predictably, self-renewal is an essential property of some cancer cells, and at least some genes that regulate normal stem-cell self-renewal also do so in cancer cells ${ }^{7-9}$. This suggests that cancers arise either from normal stem cells or from progenitor cells in which self-renewal pathways have become activated (Fig. 1b).

Using serial transplantation, Singh et al. ${ }^{1}$ demonstrated that at least some of the $\mathrm{CD}_{133}{ }^{+}$brain-cancer cells can renew themselves. By contrast, the ability of CD133cancer cells to proliferate was limited. Soon after injection of the $\mathrm{CD}_{133^{-}}$cells into the brain, they stopped growing, leaving behind a small cluster of quiescent tumour cells.

This outcome is reminiscent of the observation that many women with small clusters of metastatic breast-cancer cells in their bone marrow, who did not receive any treatment, survived for years without progression of their cancer ${ }^{10}$. One explanation for this tumour dormancy is that the microscopic clusters of cancer cells did not contain cancer stem cells and so, like the $\mathrm{CD}_{133^{-}}$braincancer cells, were unable to grow further. Taken together with the observation that circulating cancer cells in the blood are an indicator of prognosis in breast-cancer patients ${ }^{11}$, this suggests that the use of markers to reveal cancer stem cells could help in making decisions about treatments.

The identification of cancer stem cells is a significant step in the fight against these dreaded diseases: because self-renewal is essential if tumours are to grow, agents that target such cells may be effective treatments. A possible complication is that the mechanisms known to regulate cancer-stem-cell self-renewal also regulate the process in normal stem cells. Unlike normal stem cells ${ }^{12}$, however, the expansion of cancer stem cells is not tightly regulated, implying that there are significant differences between the normal and the cancerous self-renewal pathways. This gives hope that the isolation of cancer stem cells, coupled with our knowledge of the mutations causing cancer, will result in ways to eliminate cancer cells while sparing normal tissues.

Michael F. Clarke is in the Departments of Internal Medicine, and Cell and Developmental Biology, University of Michigan Medical School, CSCG Room 4410, 1500 E. Medical School Drive, Ann Arbor, Michigan 48109-0936, USA.

e-mail: mclarke@umich.edu

1. Singh, S. K. et al. Nature 432, 396-401 (2004).

2. Fialkow, P. J. Birth Defects Orig. Art. Ser. 12, 123-132 (1976).

3. Lapidot, T. et al. Nature 367, 645-648 (1994).

4. Matsui, W. et al. Blood 103, 2332-2336 (2004).

5. Al-Hajj, M., Wicha, M. S., Benito-Hernandez, A., Morrison, S. J. \& Clarke, M. F. Proc. Natl Acad. Sci. USA 100, 3983-3988 (2003).

6. Uchida, N. et al. Proc. Natl Acad. Sci. USA 97, 14720-14725 (2000)

7. Lessard, J. \& Sauvageau, G. Nature 423, 255-260 (2003).

8. Park, I. K. et al. Nature 423, 302-305 (2003).

9. van Lohuizen, M., Frasch, M., Wientjens, E. \& Berns, A. Nature 353, 353-355 (1991)

10. DiStefano, A., Tashima, C. K., Yap, H. Y. \& Hortobagyi, G. N Cancer 44, 196-198 (1979).

11. Cristofanilli, M. et al. N. Engl. J. Med. 351, 781-791 (2004).

12. Morrison, S. J. et al. J. Immunol. 168, 635-642 (2002).

\title{
Towards tomorrow's catalysts
}

\author{
Charles T. Campbell
}

The ability to predict and modify the rate-determining steps in chemical reactions would be a boon in designing better catalysts. Technical innovations in computer simulations bring that goal closer.

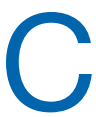
hemical conversions driven by catalysts are essential to modern society. But we must do better: sustaining industrial and economic growth, while protecting the environment, will necessitate developing more efficient processes. In particular, there is a pressing need for new solid catalysts for mixed-phase, or heterogeneous, reactions. Such reactions tend to involve large volumes, and they are the basis of, for example, more efficient ways of reducing plant and automotive emissions, and of producing cleaner fuels such as hydrogen or methanol.

A way forward lies in predicting how the details of a catalyst's surface structure control key kinetic parameters in the reaction mechanism. One such parameter is the activation barrier, which, if known for the rate-controlling elementary steps, allows the relevant rates to be calculated. These in turn enable accurate predictions of both the rate of production of the desired products and the branching ratios to undesired products, knowledge of both of which is essential to ensure a successful outcome in terms of energy efficiency and environmental impact. Writing in Physical Review Letters, Reuter et al. ${ }^{1}$ herald a powerful new approach that should ultimately help make prediction possible.

Reuter and his colleagues show just how close science has come to using $a b$ initio theoretical methods to calculate net catalytic reaction rates on complex solid surfaces. They have applied an elegant method that they call " $a b$ initio statistical mechanics", which involves two stages - first, using firstprinciples quantum mechanics to calculate the activation barriers and transition-state vibrational frequencies for all the relevant elementary surface reactions; second, to couple these through statistical mechanical methods involving transition-state theory and kinetic Monte Carlo simulations of the reaction process. Vibrational frequencies for all the intermediates and transition states, also calculated quantum mechanically, are used to incorporate entropy considerations into the calculated rate constants. Monte Carlo simulations, which are in widespread use in science, allow a highly complicated system to be sampled efficiently in a number of random configurations, the upshot being a description of the system as a whole. Kinetic Monte Carlo takes this a step further, allowing efficient sampling of the tremendous range of different timescales necessary to describe all the different elementary steps, and thus simulate the kinetics of the whole system.

In this way, Reuter et al. were able to calculate net catalytic reaction rates on solid surfaces under conditions similar to those used in industrial processes. Other workers have used a related approach to investigate different types of phenomena ${ }^{2}$, but this application to catalytic reaction rates is a particularly demanding test. Reuter et al. have achieved impressive accuracy in calculating the steady-state rates of the catalytic oxidation of carbon monoxide (a reaction performed by the catalytic converters of automobiles) over a model ruthenium oxide $\left(\mathrm{RuO}_{2}\right)$ catalyst. Their rates agree almost perfectly with excellent experimental rate measurements, performed by a different group at the same institute ${ }^{3}$, over a wide range of reaction conditions. The beauty of Reuter and colleagues' method is that it allows one to identify which elementary steps control the reaction rates and how their rates are affected by reaction conditions and, in principle, by surface structure. This is just what is needed to guide the development of better catalysts.

There is, unfortunately, one major limitation associated with the accuracy of quantum-mechanical calculations when applied to chemisorbed species on surfaces. The quantum method used by Reuter et al. is a version of density functional theory (DFT) that achieves nearly state-of-the-art accuracy in predicting the energies of such systems. Nevertheless, the authors admit that this can still be off by as much as $30 \mathrm{~kJ}$ per mol in estimating activation barriers. Given this drawback, it is surprising that they were able to achieve such impressive accuracy in their calculated carbon monoxide oxidation rates over $\mathrm{RuO}_{2}$. They attribute this agreement with experimental rates to an effect they imply is generic to catalytic reactions - the combined action of many elementary steps that simultaneously affect the rate.

Although I agree that surface-catalysed reactions generally have many elementary 